\title{
Assessment of the Seric Calcium and Parathormone Levels in Patients Underwent Hemithyroidectomy.
}

Isabel Fernández Palop ( $\square$ isabelfpalop@gmail.com )

Hospital de Sagunto y C E: Hospital de Sagunto https://orcid.org/0000-0002-1581-9436

Cristina Fernández Martínez

Hospital de Sagunto y C E: Hospital de Sagunto

María Jesús Segura Giménez

Hospital de Sagunto y C E: Hospital de Sagunto

María del Carmen Azorín Samper

Hospital de Sagunto y C E: Hospital de Sagunto

Rafael García Fuster

Universidad Catolica de Valencia San Vicente Martir

Research

Keywords: Hypocalcemia, Hemithyroidectomy, Intraoperative PTH

Posted Date: January 28th, 2021

DOI: https://doi.org/10.21203/rs.3.rs-154032/v1

License: (9) This work is licensed under a Creative Commons Attribution 4.0 International License. Read Full License 
ASSESSMENT OF THE SERIC CALCIUM AND PARATHORMONE LEVELS IN PATIENTS UNDERWENT HEMITHYROIDECTOMY.

\section{AUTHORS:}

- Isabel Fernández Palop.

Escuela de Doctorado. Universidad Católica de Valencia San Vicente Mártir.

- Cristina Fernández Martínez

Hospital de Sagunto

- María Jesús Segura Giménez

Hospital de Sagunto

- María del Carmen Azorín Samper

Hospital de Sagunto

- Rafael García Fuster

Escuela de Doctorado. Universidad Católica de Valencia San Vicente Mártir.

\section{CORRESPONDING AUTHOR}

- Isabel Fernández Palop: isabelfpalop@gmail.com 


\begin{abstract}
:
Background and objective: Hypocalcemia is one of the main complications that patients undergoing thyroid surgery can suffer. We believe that there may be an impact on serum parathormone and calcium values, despite only one thyroid cell is manipulated in thyroid lobectomy. The former explanation is the reason because we carried out a measurement and analised a potential correlation between them. Methods: This is a prospective study of 53 patients underwent thyroid lobectomy. The serum parathormone value was determined in the preoperative period, 15 min after extraction of the surgical piece, 24 hours and 3 weeks after surgery. Ionic calcium was also measured at 6 hours, 24 hours and 3 weeks. Postoperative parathormone values were compared as well as their gradient descent respect to the postoperative calcium value. The appearance of the parathyroid glands was described during surgery. Results: In none of the patients did the ionic calcium drop below $4 \mathrm{mg} / \mathrm{dl}$. The decrease in preoperative calcemia compared with the values at 6 and 24 hours had statistical significance, there were no differences compared to 3 weeks experiment. An analogous trend was observed between the percentage values of decrease in parathormone and postoperative calcemia.

Conclusions: Although the calcemia decreased after a lobectomy, it always remained above $4 \mathrm{mg} / \mathrm{dl}$. We conclude that hemithyroidectomy is a safe technique regarding the appearance of hypocalcemia in the postoperative period.
\end{abstract}

Keywords: Hypocalcemia; Hemithyroidectomy; Intraoperative PTH 


\section{BACKGROUND}

Thyroid lobectomy or hemithyroidectomy is a surgical procedure that consists in the exeresis of one of the thyroid lobes along with the isthmus, preserving the contralateral lobe. Indications for this procedure include: unilateral symptomatic goiter, toxic adenoma or study of thyroid nodules with suspicious or undetermined characteristics in fine needle aspiration cytology (1).

The advantage of this technique is that it keeps thyroid tissue functioning, thus theoretically avoiding substitution therapy in these patients, in addition to reducing the risk of complications in the contralateral lobe.

One of the most frequent complications caused after thyroid surgery is post-surgical hypoparathyroidism and hypocalcemia.

Parathyroid hormone $(\mathrm{PTH})$ is one of the three main hormones that modulate calcium and phosphate homeostasis; the other two are: calcitriol (1,25-dihydroxyvitamin D) and fibroblast growth factor 23 (FGF23) (2). Minute-by-minute control of serum ionized calcium concentration is exclusively mediated by $\mathrm{PTH}$, maintaining the concentration of this divalent cation within a narrow range by stimulating renal tubular resorption and bone resorption $(3,4)$.

PTH also stimulates the conversion of calcidiol (25-hydroxyvitamin D) into calcitriol in renal tubular cells, thus stimulating intestinal absorption of calcium and bone turnover. Calcitriol feeds back to inhibit PTH secretion indirectly through its calcemic action, as well as by exerting a direct inhibitory action on PTH biosynthesis and parathyroid cell proliferation.

Parathormone is an 84-amino acid peptide that circulates in at least four molecular forms. In normal individuals, complete, intact parathormone (amino acids 1 to 84 ) has a half-life of approximately 5 minutes.

Unlike calcium values that remain stable for a longer time, parathormone (PTH) has a short half-life, so the decrease in the amount of PTH released into the plasma means that the circulating amount of PTH decreases rapidly.

When a surgical injury to the parathyroid glands occurs, insufficient secretion of PTH occurs and consequently hypocalcemia develops. Hypocalcemia due to hypoparathyroidism can be associated with a spectrum of clinical manifestations, ranging from few symptoms to seizures, heart failure or laryngospasm, if severe.

Although this type of complication is well documented in the literature, we found wide differences in reports of incidence. Incidence varies from $0.3 \%$ to $60 \%$ in some series $(5,6)$.

Hypocalcemia due to hypoparathyroidism occurs, in most cases without documented removal of the parathyroid glands. This fact implies that these glands are highly sensitive to the injury caused by surgical manipulation (ischemia, venous congestion...). In the case of thyroid lobectomies, we assume that there will be no change in the calcification when only one thyroid cell is surgically manipulated, but work to document this fact is scarce. 
In some studies, a higher incidence of hypoparathyroidism in total thyroidectomies compared to lobectomies or subtotal thyroidectomies was found $(7,8)$.

The present work is based on the hypothesis that the manipulation in one of the two thyroid cells can have some repercussion in the release of PTH and as a consequence in the calcemia, although these variations do not cause clinical manifestations.

The objectives are:

To carry out a measurement between the concentration of PTH before and after thyroid lobectomy and to evaluate if there is a decrease between both values

Perform a measurement between the concentration of calcium ions before and after thyroid lobectomy and assess whether there is a decrease between the two values

Evaluate possible correlations between both values

\section{MATERIAL AND METHODS}

A prospective observational study was conducted including patients who underwent hemihyroidectomy at our center between February 2016 and March 2019. The study was reviewed and approved by the Center's Ethics Committee. Patients with coexisting parathyroid or renal pathology were excluded.

A consecutive sampling was carried out, collecting the characteristics of the patients and the pathology, the characteristics of the surgery, the analytical values and their evolution.

The following variables were recorded: Sex, preoperative diagnosis, thyroid function, definitive pathological anatomy; presence of symptoms and signs of hypocalcemia and other complications (recurrent paralysis, seroma, hematoma...).

As for the parathyroid glands, a description of those that could be identified, their number, condition and coloring at the end of the surgery was collected.

A blood sample was taken before the operation to determine the basal levels of ionic calcium, phosphorus and intact PTH.

We performed the measurements in ionized calcium as some studies have shown that these are more reliable than total calcium measurements in the immediate and long-term follow-up of patients with thyroidectomy (9)

An intraoperative PTH measurement was performed immediately after thyroid lobectomy (15 minutes after the surgical piece was removed). The Percentage decrease of PTH between the preoperative value and after surgery was calculated immediately:

(PTHpostop- PTHpreop) x100

PTHpreop 
A post-operative PTH determination was made 24 hours and 3 weeks after surgery.

Ionic calcium and phosphorus measurements were obtained at 6 hours, 24 hours and 3 weeks after surgery.

Ionic calcium below $4.4 \mathrm{mg} / \mathrm{dl}$ was considered hypocalcemia. In patients with symptomatic hypocalcemia or with ionic calcium values equal to or less than $4 \mathrm{mg} / \mathrm{dl}$, intravenous calcium, oral calcium, oral vitamin $\mathrm{D}$, or a combination was administered.

Statistical analysis was performed using SPSS version 22 for Windows (Chicago, IL, USA)

For the determination of the ionized calcium concentration, direct potentiometry was performed with a calcium ion-selective electrode combined with an external reference electrode, in Radiometer ABL-835 gasometer. The ionized calcium value was corrected to physiological $\mathrm{pH}$ of 7.4 .

Intact PTH was determined with the Cobas E801 analyzer from Roche Diagnostics by electrochemical luminescence enzyme immunoassay (EQL) analysis.

\section{RESULTS}

A total of fifty-three hemithyroidectomy patients were operated on between February 2016 and March 2019. These were operated on by the same team of surgeons.

$71.7 \%$ were women and $28.3 \%$ were men. In $52.8 \%$ of the cases a left hemithyroidectomy was performed and in $47.2 \%$ a right hemithyroidectomy was performed.

The surgical indication was in $43.3 \%$ of the patients for follicular proliferation, $37.7 \%$ for nodule enlargement, $11.3 \%$ for compressive symptoms and in a smaller percentage for hyperfunction or recurrent cyst.

The definitive anatomopathological diagnosis was $47.2 \%$ hyperplasia, $37.7 \%$ follicular adenoma, $7.5 \%$ Hashimoto's thyroiditis and 7.5\% thyroid carcinoma.

The targeted carcinomas were all four cases of papillary type and size less than $5 \mathrm{~mm}$. In all cases the finding of malignancy was incidental.

In one of the patients there was recurrent paralysis (1.8\%), which was transitory and recovered in less than 3 months.

Preoperative mean calcemia was $4.8 \mathrm{mg} / \mathrm{dl}(\mathrm{SD}=0.15)$; at 6 hours the mean was $4.52 \mathrm{mg} / \mathrm{dl}(\mathrm{SD}=0.2)$; at $24 \mathrm{~h}: 4.5 \mathrm{mg} / \mathrm{dl}(\mathrm{SD}=0.2)$ and at 3 weeks: $4.8 \mathrm{mg} / \mathrm{dl}(\mathrm{SD}=0.15)$.

The mean phosphatemia was: $3.35 \mathrm{mg} / \mathrm{dl}$, preoperative $(\mathrm{SD}=0.45) ; 3.38 \mathrm{mg} / \mathrm{dl}$ at $6 \mathrm{~h}$; $3.10 \mathrm{mg} / \mathrm{dl}$ at $24 \mathrm{~h}$ and $3.4 \mathrm{mg} / \mathrm{dl}$ at 3 weeks.

PTH showed a statistically significant decrease between preoperative measurement (mean: $53.84 \mathrm{pg} / \mathrm{ml}$ ), at 15 minutes (mean: $45.33 \mathrm{pg} / \mathrm{ml}$ ) and the day after surgery (mean: 
$42 \mathrm{pg} / \mathrm{ml}$ ). There was no difference between preoperative PTH and 3 weeks later (mean: $50.33 \mathrm{pg} / \mathrm{ml})$

We have not been able to find any correlation between post-operative PTH levels and calcium levels 6 hours after surgery.

We compared the percentage decrease of PTH with the ionic calcium values at 6 hours after surgery, observing that both tend to have an analogous trend. The trend line is downward, although R2 is not statistically significant. (Figure 1)

Since the number of patients in the sample is 53 and when performing a hemithyroidectomy we have to take into account the two parathyroid glands of the lobe being removed, the total number of exposed parathyroid glands would be 106.

Two parathyroid glands were identified during the procedure in 21 patients (39.6\% of cases); a single gland in 28 patients $(45.3 \%)$ and in 4 of the patients no parathyroid was identified (7.5\%).

The following diagram explains the characteristics of the glands targeted in the three groups (Figure 2)

In two of the cases, the presence of a parathyroid gland was observed in the final piece.

A calcium and PTH analysis was performed in each of these groups:

In the group in which the two parathyroid glands described were normal, the average calcification after surgery was 4.56 (SD 0.2) and the PTH 40.67. In the group in which one of the parathyroids was described as ischemic or congestive, the mean calcemia after surgery was 4.46 (SD 0.2) and the PTH 48. In the group in which one parathyroid gland was objected to in the definitive piece, the mean calcemia after surgery was 4.33 (SD 0.3) and the PTH 47.7.

We observe that the mean calcemia after hemihyroidectomy is higher in patients who have preserved both parathyroid glands and have been objectified as normal, without this being statistically significant. On the other hand, we do not observe this relationship with variations in PTH among the groups mentioned.

\section{DISCUSSION.}

Hypocalcemia post thyroidectomy as a multifactorial phenomenon is the most frequent clinical problem in patients undergoing thyroid surgery. Among the risk factors described are: extension of the resection, group IV lymph node dissection, thyroidectomy as hyperthyroid therapy, central ligation of the lower thyroid artery, number of parathyroids identified and preserved in the operative act, and the surgeon's experience (10)

The differences between preoperative and 6- and 24-hour calcemia reached statistical significance. 
This corroborates the working hypothesis that manipulation in a thyroid cell has an impact on postoperative ion calcium concentrations, although this decrease is small and recovers early by returning to baseline values at three weeks.

There are no significant differences between preoperative and three-week calcium. There are no significant differences between 6- and 24-hour calcemias, so one of the two measurements would be sufficient to document patient calcemia.

Mean phosphatemia was: $3.35 \mathrm{mg} / \mathrm{dl}$, preoperative $(\mathrm{SD}=0.45) ; 3.38 \mathrm{mg} / \mathrm{dl}$ at $6 \mathrm{~h}$; $3.10 \mathrm{mg} / \mathrm{dl}$ at $24 \mathrm{~h}$ and $3.4 \mathrm{mg} / \mathrm{dl}$ at 3 weeks. There is no significant variation between these values.

PTH showed a statistically significant decrease between preoperative measurement (mean: $53.84 \mathrm{pg} / \mathrm{ml}$ ), at 15 minutes (mean: $45.33 \mathrm{pg} / \mathrm{ml}$ ) and the day after surgery (mean: $42 \mathrm{pg} / \mathrm{ml}$ ). This fact once again confirms our hypothesis about the impact of surgery on the parathyroid glands. This repercussion can be documented immediately. This fact is well known thanks to pathologies such as primary hyperparathyroidism. The measurement of intraoperative turbo PTH allows to confirm the removal of a parathyroid adenoma thanks to the decrease in serum PTH concentration that occurs almost immediately thanks to the short half-life of the hormone.

We have documented that the performance of lobectomy also induces a decrease in serum PTH concentration even though it remains in the normal range.

There was no difference between preoperative PTH and 3 weeks later (mean: $50.33 \mathrm{pg} / \mathrm{ml}$ ), since the two contralateral glands remain intact in any case.

The fact that we found no correlation between postoperative PTH levels and postoperative calcium levels may be related to the fact that there is no postoperative hypocalcemia in the sample. However, we have found that the decrease in postoperative PTH follows a trend analogous to calcium levels.

The absence of differences between the calcemias at 6 and 24 hours would allow us to obviate the practice of the latter. The six-hour calcemia predicts what the 24-hour level will be, if we need to objectify this data. This may be of interest given the current extent of the WFS.

We understand that thyroid lobectomy may be an ideal procedure to perform in a Major Outpatient Surgery (surgical procedures of medium complexity for which the patient is admitted to the hospital on the day of the operation and is allowed to return home the same day). The most serious complications (bleeding and recurrent paralysis) appear in the first hours after surgery $(11,12)$. In the case of thyroid lobectomy, the possible paralysis or paresis would be unilateral, causing dysphonia, but not severe problems such as dyspnea or respiratory failure. Our work allows us to guarantee the safety of the ambulatory surgery with respect to possible hypocalcemia: On the one hand, the drops in serum calcium recorded do not reach hypocalcemia figures, and on the other hand, we can predict it in only six hours, this measurement being comparable to the one we would take after 24 hours. 
The results of the study allow us to corroborate that one of the factors influencing the development of hypocalcemia is the extent of thyroidectomy.

\section{CONCLUSIONS:}

The data from the study indicate that there are no significant differences between preoperative and postoperative calcemia performed.

The absence of differences between calcium at six and 24 hours, ensures that if the calcemia is normal at six hours postoperatively, there will be no hypocalcemia in the subsequent hours, which allows us to perform lobectomy in outpatient surgery since hypocalcemia is not a complication expected after discharge from hospital.

Patients who undergo this type of surgery can be discharged early and without replacement treatment with calcium or vitamin D.

Our results showed that performing a thyroid lobectomy is a safe intervention, in which no post-operative calcemias (ionic calcium) below $4 \mathrm{mg} / \mathrm{dl}$ have been obtained.

We have observed a decrease between pre and intraoperative PTH even though both are in the normal range.

As for the measurement of the calcemia, the minimal deviations in the ionic calcium averages at different times, indicate the reliability of this technique for the control and follow-up of the patients.

\section{LIST OF ABBREVIATIONS}

- PTH: Parathyroid hormone 


\section{DECLARATIONS:}

The authors declare that the study was reviewed and approved by the Ethics Committee of Sagunto Hospital. All patients included in the study signed a specific consent form to use their data.

The datasets used and analysed during the current study are available from the corresponding author on reasonable request.

The authors declare that they have no competing interests.

All authors have contributed intellectually to the work, are eligible for authorship and have approved the final version of the work. On their behalf, I declare that the work is original, is not published already in part or whole in any journal or magazine for private or public circulation and is not under consideration of publication elsewhere. 


\section{REFERENCES:}

1. Haugen BR, Alexander EK, Bible KC, Doherty GM, Mandel SJ, Nikiforov YE, et al. 2015 American Thyroid Association Management Guidelines for Adult Patients with Thyroid Nodules and Differentiated Thyroid Cancer: The American Thyroid Association Guidelines Task Force on Thyroid Nodules and Differentiated Thyroid Cancer. Thyroid : official journal of the American Thyroid Association. 2016 Jan;26(1):1-133. PubMed PMID: 26462967. Pubmed Central PMCID: 4739132.

2. Kumar R, Thompson JR. The regulation of parathyroid hormone secretion and synthesis. Journal of the American Society of Nephrology : JASN. 2011 Feb;22(2):216-24. PubMed PMID: 21164021. Pubmed Central PMCID: 5546216.

3. Pak CY. Calcium metabolism. Journal of the American College of Nutrition. 1989;8 Suppl:46S-53S. PubMed PMID: 2681327.

4. Brown EM. Four-parameter model of the sigmoidal relationship between parathyroid hormone release and extracellular calcium concentration in normal and abnormal parathyroid tissue. The Journal of clinical endocrinology and metabolism. 1983 Mar;56(3):572-81. PubMed PMID: 6822654.

5. Testini M, Gurrado A, Lissidini G, Nacchiero M. Hypoparathyroidism after total thyroidectomy. Minerva chirurgica. 2007 Oct;62(5):409-15. PubMed PMID: 17947951.

6. Rosato L, Avenia N, Bernante P, De Palma M, Gulino G, Nasi PG, et al. Complications of thyroid surgery: analysis of a multicentric study on 14,934 patients operated on in Italy over 5 years. World journal of surgery. 2004 Mar;28(3):271-6. PubMed PMID: 14961204.

7. Pelizzo MR, Bernante P, Toniato A, Piotto A, Grigoletto R. [Hypoparathyroidism after thyroidectomy. Analysis of a consecutive, recent series]. Minerva chirurgica. 1998

Apr;53(4):239-44. PubMed PMID: 9701977. Ipoparatiroidismo dopo tiroidectomia. Verifica di una serie consecutiva e recente.

8. Wilson RB, Erskine C, Crowe PJ. Hypomagnesemia and hypocalcemia after thyroidectomy: prospective study. World journal of surgery. 2000 Jun;24(6):722-6. PubMed PMID: 10773126.

9. Tartaglia F, Giuliani A, Sgueglia M, Patrizi G, Di Rocco G, Blasi S, et al. Is ionized calcium a reliable predictor of hypocalcemia after total thyroidectomy? A before and after study. Il Giornale di chirurgia. 2014 Jan-Feb;35(1-2):27-35. PubMed PMID: 24690338.

Pubmed Central PMCID: 4321580.

10. Thomusch O, Machens A, Sekulla C, Ukkat J, Brauckhoff M, Dralle H. The impact of surgical technique on postoperative hypoparathyroidism in bilateral thyroid surgery: a multivariate analysis of 5846 consecutive patients. Surgery. 2003 Feb;133(2):180-5. PubMed PMID: 12605179.

11. Harding J, Sebag F, Sierra M, Palazzo FF, Henry JF. Thyroid surgery: postoperative hematoma--prevention and treatment. Langenbeck's archives of surgery. 2006 Jun;391(3):16973. PubMed PMID: 16555087.

12. Edis AJ. Prevention and management of complications associated with thyroid and parathyroid surgery. The Surgical clinics of North America. 1979 Feb;59(1):83-92. PubMed PMID: 582079. 

Figures

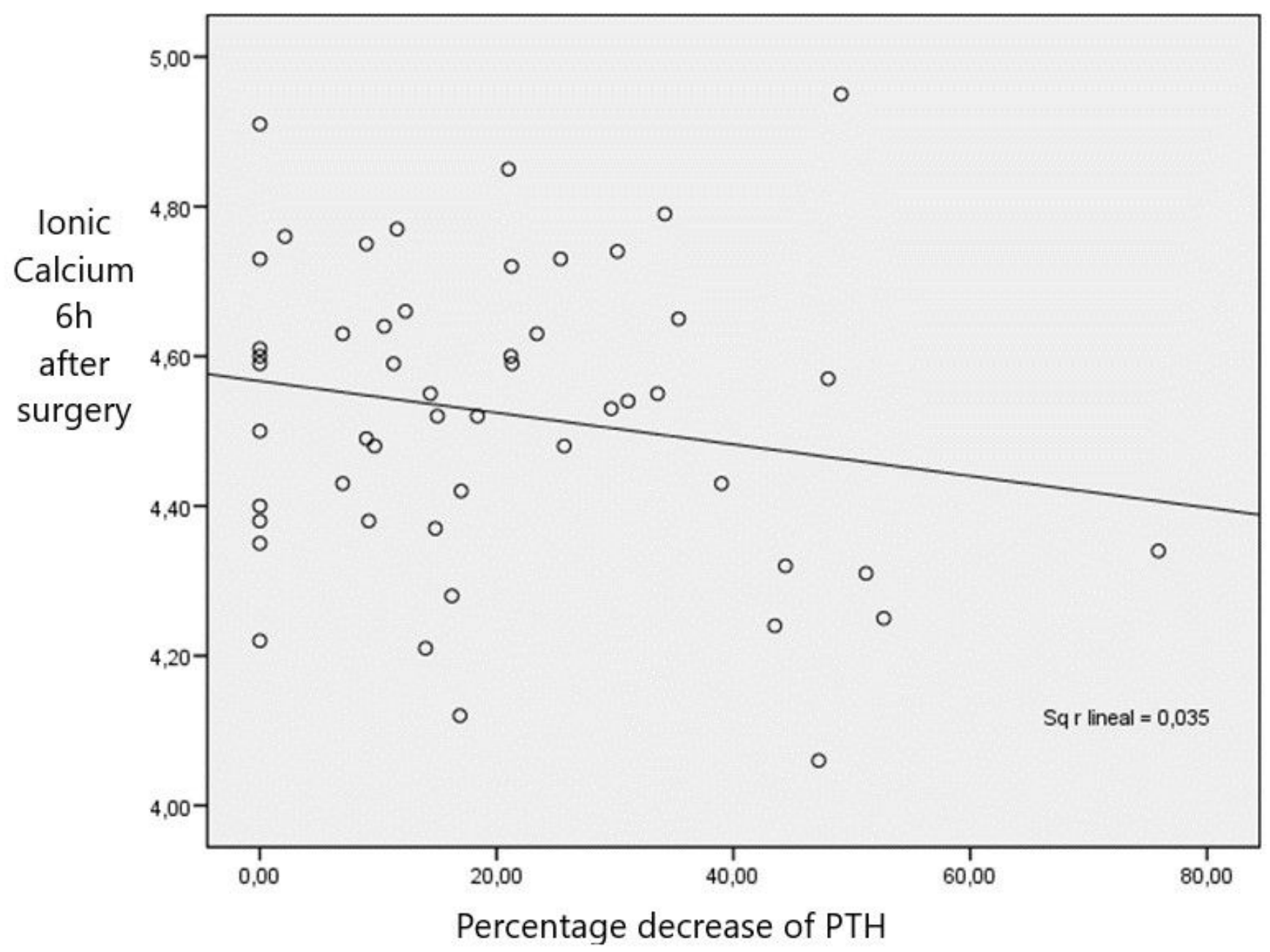

\section{Figure 1}

Pearson's correlation for percentage decrease in PTH and calcemia obtained 6 hours after the intervention. 


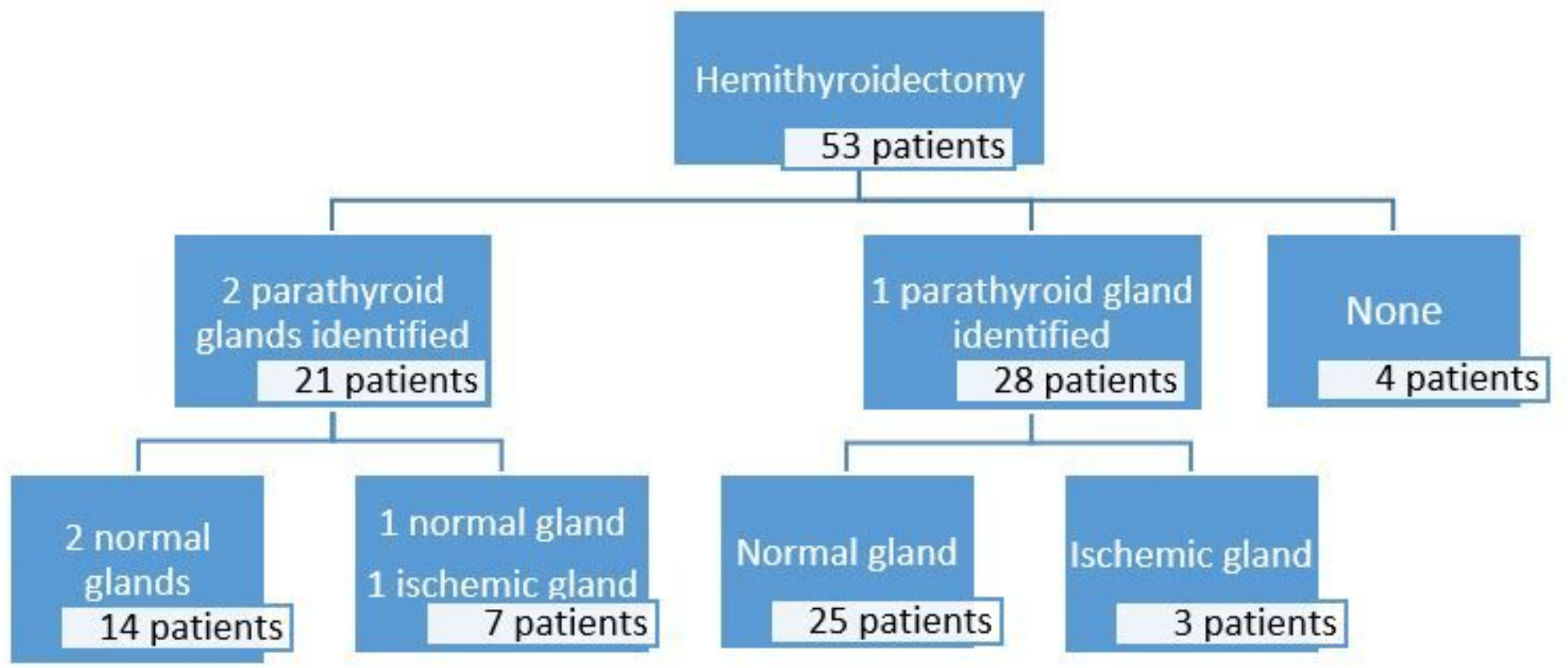

Figure 2

Parathyroid glands identified in surgery and their characteristics 